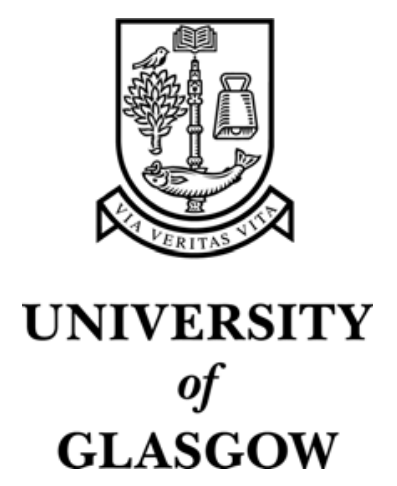

Brewster, S.A. (1998) Using earcons to improve the usability of tool palettes. In, Conference on Human Factors in Computing Systems, 18-23 April 1998, pages pp. 297-298, Los Angeles, California, USA.

http://eprints.gla.ac.uk/3240/ 


\title{
Using Earcons to Improve the Usability of Tool Palettes
}

\author{
Stephen A Brewster \\ Glasgow Interactive Systems Group, Department of Computing Science \\ University of Glasgow, Glasgow, G12 8QQ, UK \\ Tel: +44 (0)141330 4966, Fax: +44 (0)141330 4913 \\ Email: stephen@dcs.gla.ac.uk, Web: www.dcs.gla.ac.uk/ stephen/
}

\begin{abstract}
This paper describes an experiment to investigate the effectiveness of adding sound to tool palettes. Palettes have usability problems because users need to see the information they present but they are often outside the area of visual focus. Non-speech sounds called earcons were used to indicate the current tool and tool changes so that users could tell what tool was in use, wherever they were looking. Experimental results showed a significant reduction in the number of tasks performed with the wrong tool. Users knew what the current tool was and did not try to perform tasks with the wrong one.
\end{abstract}

\section{INTRODUCTION}

Tool (or button) palettes are a common feature of most graphical interfaces. They allow the user access to a set of tools and indicate which one is active. Figure 1 (a) shows a set of tools from a graphics package, where palettes are common. The currently selected tool (in this case the rectangle tool) is highlighted by changing its border.

In some systems (e.g. Microsoft Word's drawing package) after one rectangle has been drawn the system changes back to the default tool, often the selection tool (the dotted square at the top left in Figure 1 (a)). In other systems (e.g. Adobe Illustrator) the tool will remain permanently selected until the user changes it. There is a hybrid of these (e.g. ClarisDraw) where the user can single-click a tool for it to be selected once or double-click for it to be permanently selected. This method has the advantage that users can choose whether they want to stay in a drawing tool or revert back to the selection tool - it is more flexible. Figure 1 (a) shows an example of the feedback indicating a single click and (b) shows a double click.

Interaction problems occur because users may not notice the currently active tool. In a graphics package users will be occupied with the drawing task they are doing (e.g. drawing a series of rectangles) which will require their full visual attention. This means that they will not be looking at the palette to see the current tool. If the system switches back to the default tool users may try to draw another rectangle but end up using the selection tool by mistake (or vice versa). These problems are exacerbated by the hybrid system because it is less predictable - the user may not remember if the current tool was single or double clicked.

To solve the problems of mis-selection users must get the right feedback. In this paper it is suggested that using sound can solve the problems. Why use sound, and not graphical feedback? It is difficult to solve these problems with extra graphics. Graphics displayed on the palette will not be seen by users as their attention will be on the drawing task they are engaged in. The human visual system has a narrow area of focus which means that users cannot look at the palette as well as their main task [6]. Information could be displayed at the mouse location often the shape of the cursor is changed to reflect the current tool. This has some effect but if the cursors are too big they will obscure the drawing underneath or if they are too small they will be too subtle to be noticed. Nonspeech sound has many advantages: It does not take up any screen space, it is good at getting attention and it does not disrupt our visual focus.

Although there has been no other work on the use of sound in palettes, there has been some on the use of sound to improve other graphical widgets. Brewster and colleagues have successfully improved the usability of buttons, scrollbars and menus with sound [4, 6]. They reduced the time taken to recover from errors, to complete tasks, and workload without any increase in annoyance. Beaudouin-Lafon et al. [1] added sound to solve usability problems in scrollbars. They used an auditory illusion called Shepard-Risset tones which increase (or decrease) in pitch indefinitely. When the user was scrolling down a continuously decreasing tone was used, when scrolling up an increasing one. If scrolling errors occurred then the user would hear tones moving in the wrong direction. Results from these studies suggested that sound would be effective in solving the problems with palettes.

\section{EXPERIMENT}

An experiment was needed to see if the addition of sound could solve the problems of tool mis-selection. Twelve participants were used (undergraduate students from the University of Glasgow). The main hypotheses were:

The auditory feedback should make the task easier because participants will be able to tell when tool changes occur. There should be no increase in annoyance due to the sounds as they will be providing information that the participants need to overcome usability problems. The number of tasks performed with the wrong tool should be reduced as users will know which tool is currently active. (a)

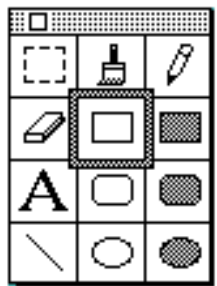

(b)

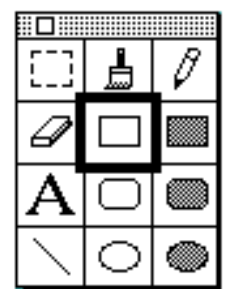


The experiment was a counterbalanced, two-condition, repeated-measures, within-subjects design. Each participant used both the standard and auditory tool palettes. During each condition the participants had to perform standard drawing tasks set by the experimenter. To get a full measurement of usability error rates, annoyance and user preference were measured [2].

Participants were required to perform drawing tasks set by the experimenter in a simple graphics package. The package was a standard one with standard tools (the tool palette used is shown in Figure 1). The tools were differentiated by cursor shape using the standard Macintosh cursor shapes. The hybrid method of tool selections was implemented in the package.

The drawing tasks performed involved users drawing a car, a tree and a sun. The eight car-drawing tasks were described step-by-step, the final two tasks were left open. The tasks were designed to mimic the standard drawing tasks a user might perform. The tasks also provided the opportunity for single and double clicking the tools.

The sounds used were based around structured non-speech audio messages called Earcons [3, 5] and were created using the earcon design guidelines [5, 7].

The main problems with tool palettes occur when switching from one tool to another. If the user does not know a switch has occurred (or does not know that the same tool is still active) then errors will result. Therefore, an earcon was played when a new tool was chosen. This occurred when (a) the user clicked on a new tool in the palette or (b) after he/she had finished drawing an object. In (a) this could be a single- or double-click sound. In (b) if no tool change occurred the same tool earcon was played again to reinforce that the tool had not changed, otherwise a sound indicating a switch back to the default tool was played. The default selection tool had a marimba timbre and the other tools a trumpet timbre. Only two instruments were needed because any automatic tool changes would always be from a drawing tool to the default tool.

For a single-click selection one $100 \mathrm{msec}$. note at pitch $\mathrm{C}_{3}$ was played. When a tool was selected by a double click the user heard the single-click earcon, to indicate a change in tool, and then two 100 msec. notes at a higher pitch, $\mathrm{C}_{2}$, to indicate a double-click selection. These sounds were played in the timbre of the tool selected.

\section{RESULTS}

There was no significant difference in annoyance between the conditions $\left(T_{11}=0.24, \mathrm{p}=0.81\right)$. Six of the participants felt the visual condition was more annoying, five felt the auditory more annoying and one felt them equal. There was no significant difference in overall preference $\left(T_{11}=1.70, \mathrm{p}=0.12\right)$. Nine participants preferred the sonic tool palette, three preferred the standard, graphical one.

Figure 2 shows the number of tasks performed with the wrong tool. There was a significant reduction in the auditory condition $\left(T_{11}=3.08, \mathrm{p}=0.01\right)$. In total, eight participants never used a wrong tool in the auditory condition with only three in the visual. The average number of tasks performed with the wrong tool fell from 3.25 in the visual condition to 0.83 in the auditory condition. This indicated that participants noticed they

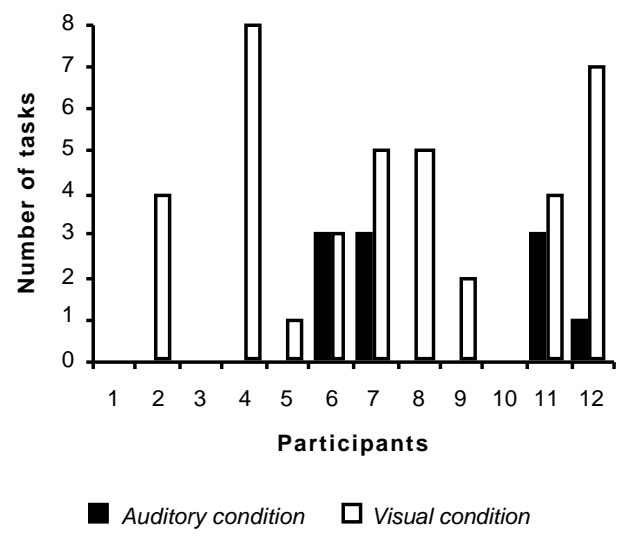

Figure 2: Number of tasks performed with wrong tool.

were in the wrong tool and, in most cases, changed to the correct tool straightaway.

\section{CONCLUSIONS}

The results showed no difference in the annoyance experienced by users. This indicated that if care was taken in the design of the earcons, and they solve specific usability problems, users will find them useful and not annoying. This confirms our previous findings [4, 6].

There were significantly fewer tasks performed with the wrong tool in the auditory condition. This meant the earcons were indicating what tool was in use and users did not forget. In fact only four of the twelve participants tried to perform any of the tasks with the wrong tools in the auditory condition. When the system changed back to the default tool automatically errors were likely; Users might not be expecting a change. The results showed that the auditory feedback did indicate this to users significantly better than the standard graphical feedback. Therefore, the earcons were effective at indicating tool changes.

\section{REFERENCES}

1. Beaudouin-Lafon, M. \& Conversy, S. (1996). Auditory illusions for audio feedback. In ACM CHI'96 Conference Companion, Vancouver, Canada: Addison-Wesley, pp. 299-300.

2. Bevan, N. \& Macleod, M. (1994). Usability measurement in context. International Journal of Man-Machine Studies, 13(1 \& 2), pp. 123-145.

3. Blattner, M., Sumikawa, D. \& Greenberg, R. (1989). Earcons and icons: Their structure and common design principles. Human Computer Interaction, 4(1), pp. 1144.

4. Brewster, S.A. (1997). Using Non-Speech Sound to Overcome Information Overload. Displays, 17, pp. 179-189.

5. Brewster, S.A., Wright, P.C. \& Edwards, A.D.N. (1993). An evaluation of earcons for use in auditory human-computer interfaces. In Proceedings of ACM INTERCHI'93, Amsterdam, Holland: Addison-Wesley, pp. 222-227.

6. Brewster, S.A., Wright, P.C. \& Edwards, A.D.N. (1994). The design and evaluation of an auditoryenhanced scrollbar. In Proceedings of ACM CHI'94, Boston, MA: Addison-Wesley, pp. 173-179.

7. Brewster, S.A., Wright, P.C. \& Edwards, A.D.N. (1995). Experimentally derived guidelines for the creation of earcons. In Adjunct Proceedings of $B C S$ HCI'95, Huddersfield, UK, pp. 155-159. 